\title{
UNA PARADOJA EN \\ LA MÁXIMA QUINEANA DE QUE NINGÚN ENUNCIADO ES INMUNE A LA REVISIÓN
} Emilio Méndez Pinto*

RESUMEN: En este trabajo se muestra que, si se lleva a su conclusión lógica la máxima de Quine, "Ningún enunciado es inmune a la revisión" (es decir, a que la propia máxima quineana no es inmune a la revisión), desemboca una situación paradójica. Tal paradoja es, no obstante, inofensiva para la máxima de que "Ningún enunciado es inmune a la revisión" porque no la autorrefuta.

PALABRAS ClAVE: Quine, analiticidad, sinteticidad, enunciados autorrefutables, enunciados refutables.
THE PARADOX OF QUINE'S ADAGE

"NO STATEMENT IS IMMUNE TO REVISION"

ABSTRACT: In this article, we will show that if Quine's adage is fulfilled "No statement is immune to revision" (that is, that the adage is not immune to revision), it results in a paradoxical situation. Such paradox is nonetheless harmless to the adage "No statement is immune to revision" because it is undeniable.

KEYWORDS: Quine, analyticity, syntheticity, undeniable statements, deniable statements. 


\author{
UNA PARADOJA EN \\ LA MÁXIMA QUINEANA \\ DE QUE NINGÚN ENUNCIADO \\ ES INMUNE A LA REVISIÓN
}

La máxima es esta: "Ningún enunciado es inmune a la revisión". ${ }^{1}$ De esta máxima se sigue que para cada enunciado hay circunstancias en las que su rechazo sería racional. En Mente y cuerpo, Putnam ${ }^{2}$ sostiene que el enunciado "Ningún enunciado es inmune a la revisión" es, si se contempla lo que de él se sigue, falso: en ninguna circunstancia sería racional rechazar el enunciado "No

${ }^{1}$ W. V. O. Quine, "Two dogmas of empiricism", en W. V. O. Quine, From a logical point of view. Nine logico-philosophical essays, 1980, Harvard, Harvard University Press, p. 43.

${ }^{2}$ Hilary Putnam, "Mente y cuerpo", en Hilary Putnam, Razón, verdad e historia, 2006, Madrid, Tecnos, p. 90, en donde el argumento está expuesto tal como se encuentra aquí.

${ }^{3}$ Aquí consideraremos a la máxima de Quine como un enunciado más, es decir, como un miembro del conjunto de todos los enunciados. todos los enunciados son verdaderos", es decir, aceptar el enunciado "Todos los enunciados son verdaderos". No es difícil imaginar casos para los que el tipo de réplica de Putnam a la máxima de Quine funcione exactamente igual de bien. Por ejemplo, el caso más obvio: en ninguna circunstancia sería racional rechazar el enunciado "No todos los enunciados son falsos", es decir, aceptar el enunciado "Todos los enunciados son falsos".

Ahora bien, de ninguna manera ocurre que el tipo de réplica de Putnam sea igualmente válido para todos los casos de revisión de enunciados. Ocurre, más bien, que los enunciados "No todos los enunciados son verdaderos" y "No todos los enunciados son falsos" se prestan para 
mostrar que la máxima quineana de que ningún enunciado es inmune a la revisión (o, mejor dicho, lo que se sigue de esta máxima) es irremediablemente falsa para algunos casos. Pero este tipo de réplica, incluso conservando el término "todos" en los enunciados por revisar, no es válido, por ejemplo, en las ciencias naturales. En estas no hay enunciados analiticos, y por tanto, la máxima "Ningún enunciado [de las ciencias naturales] es inmune a la revisión" es perfectamente válida.

En esta breve nota me gustaría examinar otro aspecto de la máxima quineana. Este aspecto es el siguiente:

$\mathrm{Si}$

(1) Ningún enunciado es inmune a la revisión,

el enunciado (1) no es inmune a la revisión.

Una revisión radical ${ }^{4}$ del enunciado (1) nos haría aceptar que

${ }^{4}$ Una revisión radical es, por así decirlo, una revisión que da un giro de 180 grados al sentido original del enunciado en revisión. Por ejemplo, una revisión radical del enunciado "Todos los enunciados son verdaderos" produciría, una vez llevada a cabo, el enunciado "Todos los enunciados son falsos" o "Ningún enunciado es verdadero". Una revisión radical del enunciado "Todos los enunciados son falsos" produciría, una vez llevada a cabo, el enunciado "Todos los enunciados son verdaderos" o "Ningún enunciado es falso". Por otra parte, llamaremos revisión moderada a la revisión que, si bien transforma el sentido de un enunciado, no lo hace radicalmente. Por ejemplo, una revisión moderada
(2) Cualquier enunciado es inmune a la revisión.

Entonces el enunciado (2) es inmune a la revisión.

(Desde luego, si en esta teoría de enunciados vulnerables a la revisión se rechazan las revisiones radicales, el enunciado (2) nunca se produciría. En su lugar, resultarían enunciados derivados de revisiones moderadas del enunciado (1), del tipo "Algunos enunciados son inmunes a la revisión" o "Algunos enunciados no son inmunes a la revisión", lo que equivale a decir, respectivamente, que algunos enunciados no son revisables o que algunos enunciados son revisables. Es claro que entre cualquiera de estos dos enunciados y el enunciado (1) hay una diferencia significativa. Pero también es claro que en la teoría de enunciados vulnerables a la revisión no hay ninguna razón por la cual deban rechazarse revisiones radicales en aras de

del enunciado "Todos los enunciados son verdaderos" produciría, una vez llevada a cabo, el enunciado "No todos los enunciados son verdaderos" (o, equivalentemente, "Hay al menos un enunciado falso"), del mismo modo que una revisión moderada del enunciado "Todos los enunciados son falsos" produciría el enunciado "No todos los enunciados son falsos" (o, equivalentemente, "Hay al menos un enunciado verdadero"). Por último, también puede haber revisiones que, una vez llevadas a cabo, reafirmen al enunciado en revisión (es decir, que den, por así decirlo, un giro de 360 grados al sentido del enunciado en revisión, volviendo así al mismo lugar). Podríamos llamar "revisiones analíticas" o "revisiones circulares" a tal tipo de revisiones. 
privilegiar revisiones únicamente moderadas.)

Pero también habría que revisar, si hemos de ser consistentes (coherentes), el enunciado según el cual habría que revisar el enunciado (1). Entonces el enunciado

(3) El enunciado "Ningún enunciado es inmune a la revisión" no es inmune a la revisión

no es inmune a la revisión.

Pero entonces podría suceder que, bajo una revisión radical del enunciado (3) (ya vimos que no hay nada que nos impida hacer esto), aceptemos que

(4) No es el caso que el enunciado "Ningún enunciado es inmune a la revisión" no es inmune a la revisión.

Si aceptamos (4), entonces el enunciado (1) es inmune a la revisión.

En resumen: si llevamos a cabo una revisión radical del enunciado "Ningún enunciado es inmune a la revisión", nos encontramos con que el enunciado que resulta de tal revisión (es decir, (2)) es inmune a la revisión, mientras que si llevamos a cabo una revisión radical del enunciado según el cual habría de revisarse al enunciado "Ningún enunciado es inmune a la revisión" (es decir,
(3) y su propiedad de que no es inmune a la revisión), nos encontramos con que, a partir del enunciado que resulta de tal revisión (es decir, (4)), el enunciado "Ningún enunciado es inmune a la revisión" es inmune a la revisión.

En aras de la claridad, expondré lo anterior de la manera más explícita posible.

Si a la máxima quineana de que "ningún enunciado es inmune a la revisión" la consideramos como un enunciado más (es decir, como una afirmación que tiene el predicado de ser revisable), entonces una revisión radical de tal enunciado (es decir, una revisión que da un giro de 180 grados al sentido del enunciado "Ningún enunciado es inmune a la revisión") produce el enunciado "Cualquier enunciado es inmune a la revisión" o, equivalentemente, "Ningún enunciado es revisable". Inmediatamente es claro que, según lo que indica el propio enunciado "Ningún enunciado es revisable", este es inmune a la revisión.

Ahora bien, si revisamos radicalmente al enunciado que nos permite revisar el enunciado "Ningún enunciado es inmune a la revisión" (es decir, si revisamos radicalmente "El enunciado 'ningún enunciado es inmune a la revisión' no es inmune a la revisión"), nos encontramos con que "No es el caso que el enunciado 'ningún enunciado es inmune a la 
revisión' no es inmune a la revisión". Entonces, según esto último, el enunciado "Ningún enunciado es inmune a la revisión" es inmune a la revisión.

Este aspecto que resaltamos de la máxima quineana de que ningún enunciado es inmune a la revisión difiere del aspecto destacado por Putnam para la misma máxima en el hecho obvio de que a partir de nuestro examen surge una situación paradójica, mientras que del examen de Putnam resulta que la máxima quineana no es verdadera para cualquier caso. Sin embargo, la estrategia en ambos casos es esencialmente la misma: consiste en aplicar lo propuesto por la máxima "Ningún enunciado es inmune a la revisión" a la misma máxima "Ningún enunciado es inmune a la revisión". Para el aspecto que nosotros destacamos, tal estrategia fue explícita desde el principio, pero para el aspecto destacado por Putnam, tal estrategia también está presente (aunque sea de forma implícita). Si no fuese así, es decir, si la estrategia de Putnam no fuese la de aplicar lo sugerido por la máxima "Ningún enunciado es inmune a la revisión" a la propia máxima "Ningún enunciado es inmune a la revisión”, entonces no habría llegado a la conclusión de que tal máxima es falsa (al menos no en el modo en el que lo hizo). Esto, porque el rechazo a revisar el enunciado "No todos los enunciados son verdaderos" se sigue del rechazo a aceptar la máxima "Ningún enunciado es inmune a la revisión" como analíticamente verdadera, es decir, de la admisión de que la propia máxima "Ningún enunciado es inmune a la revisión" es revisable.

\section{¿Por qué la máxima de Quine no se autorrefuta?}

A modo de conclusión para esta breve nota, vale la pena examinar por qué, a pesar de todo lo dicho, la máxima "Ningún enunciado es inmune a la revisión" no se autorrefuta. Por el contrario, creo que, justamente por todo lo dicho, de algún modo sale reforzada, es decir, de algún modo sale validada. Para mostrar esto, consideremos de nuevo nuestra estrategia. Esta consistió en aplicar al enunciado "Ningún enunciado es inmune a la revisión" lo indicado por el propio enunciado "Ningún enunciado es inmune a la revisión". Nuestro enunciado en cuestión se habría autorrefutado si lo que expresa no fuese aplicable al propio enunciado, ${ }^{5}$ es decir, si el enunciado "Ningún enunciado es inmune a la revisión" no fuese revisable por el mismo canon de que

${ }^{5}$ Obviamente, si las afirmaciones o los supuestos que contempla un enunciado $x$ no se cumplen para un enunciado $y$, pero sí para el propio enunciado $x$, entonces el enunciado $x$ se refuta, pero no se autorrefuta. 
ningún enunciado es inmune a la revisión. Como inmediatamente se ve, esto es un absurdo (sencillamente porque "Ningún enunciado es inmune a la revisión" es semánticamente equivalente a "cualquier enunciado es revisable").

Precisamente por este absurdo, de cierto modo se valida la máxima quineana: esta puede refutarse (como cuando uno considera los casos de que "No todos los enunciados son verdaderos" o de que "No todos los enunciados son falsos", que no sería racional rechazar en ninguna circunstancia), pero no se autorrefuta. (He aquí su carácter no analítico, ique es justamente la caracterización quineana de los enunciados!) Esta propiedad, la de no autorrefutarse, no la tiene, por ejemplo, una de las máximas más influyentes en la teoría del conocimiento del siglo XX: el criterio de significación del positivismo lógico. Este criterio es que "el significado de una oración es su método de verificación”, y la exposición de por qué se autorrefuta se debe, en un sentido importante, a Putnam:

En los últimos cincuenta años, la manifestación más clara de la tendencia a pensar que los métodos de "justificación racional" son otorgados por algo así como una lista o canon (si bien esos filósofos de la ciencia admiten no haber logrado aún una forma- lización plena) fue el movimiento conocido como Positivismo Lógico. Los positivistas no solo confiaban en que los "lógicos de la ciencia" (este era su término para denominar a los filósofos) lograrían dejar constancia escrita de una descripción (pretendidamente exhaustiva) del método científico, sino que, según ellos, el "método científico" agotaba la propia racionalidad, y la contrastabilidad mediante ese método agotaba la significatividad ("el significado de una oración es su método de verificación"); la lista o canon determinaría lo que tiene significación cognitiva. Los enunciados contrastables mediante métodos pertenecientes a la lista (los métodos de la matemática, la lógica y las ciencias empíricas) contarían como significativos; los positivistas mantenían que todos los demás eran sólo "pseudo-enunciados", o sinsentidos disfrazados.

Una réplica obvia consistía en afirmar que el criterio de significación del positivismo lógico se autorrefuta: el mismo criterio no es ni (a) "analítico" (un término que los positivistas utilizaban para dar cuenta de la lógica y de la matemática), ni (b) empíricamente contrastable. ${ }^{6}$

${ }^{6}$ Hilary Putnam, "Dos concepciones de la racionalidad", en Hilary Putnam, Razón, verdad e historia, p. 111. 


\section{Un ejemplo más de una máxima que se autorrefuta}

Considérese la máxima de que "El significado de un enunciado es su uso". $7 \mathrm{Si}$ el significado de un enunciado es su uso, entonces el significado del enunciado "El significado de un enunciado es su uso" es su uso. "El significado de un enunciado es su uso" quiere decir que el modo en el que se usa un enunciado condiciona su significado (claramente, no puede determinar su significado porque este está sujeto al uso, que no es uno sino múltiple). Ahora bien, si un uso específico y lingüísticamente legítimo o válido del enunciado "El significado de un enunciado es su uso" llegase a condicionar su significado ( $\sin$ llegar a determinarlo), ¿cuáles serían otros usos igualmente específicos y lingüísticamente legítimos o válidos del enunciado "El significado de un enun- ciado es su uso" que igualmente condicionarían su significado (sin llegar a determinarlo)?

Prima facie, parece que no hay una clase de casos de utilización (por más pequeña que sea) del enunciado "El significado de un enunciado es su uso" que, en distintos contextos, énfasis, intenciones, etc., condicione el significado del enunciado "El significado de un enunciado es su uso"; en otra palabras, parece que el uso del enunciado "El significado de un enunciado es su uso" es irrelevante al momento de especificar (indicar, mostrar, etc.) su significado: este es literalmente "El significado de un enunciado es su uso" indistintamente del uso del enunciado "El significado de un enunciado es su uso". Si lo anterior es correcto, entonces la máxima de que "El significado de un enunciado es su uso" se autorrefuta.

\footnotetext{
${ }^{7}$ Este tema lo considero detalladamente en Emilio Méndez Pinto, ¿Se autorrefuta el supuesto de que el significado de un enunciado es su uso?, 2016, México, La Reina de las Ciencias, Biblioteca Digital del ILCE, UNESCO. Este trabajo es una extrapolación, para el caso de los enunciados, del principio de Wittgenstein según el cual el significado de una palabra es su uso en el lenguaje. Véase Ludwig Wittgenstein, Investigaciones filosóficas, 2003, México, Instituto de Investigaciones Filosóficas, UNAM, p. 61. No obstante, de este principio de Wittgenstein no debe seguirse que Wittgenstein propuso una teoría según la cual "el significado es el uso". Para este último punto, véase Hilary Putnam, El pragmatismo. Un debate abierto, 2006, Barcelona, Gedisa, p. 23.
} 
CITAM Derechos Reservados.

La reproducción total o parcial de este artículo se podrá hacer si el ITAM otorga la autorización previamente por escrito. 